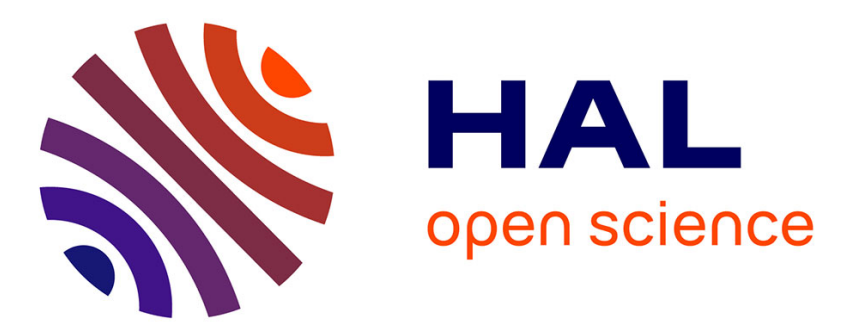

\title{
The influence of individual social traits on robot learning in a human-robot interaction
}

\author{
Hakim Guedjou, Sofiane Boucenna, Jean Xavier, David Cohen, Mohamed
}

Chetouani

\section{- To cite this version:}

Hakim Guedjou, Sofiane Boucenna, Jean Xavier, David Cohen, Mohamed Chetouani. The influence of individual social traits on robot learning in a human-robot interaction. 2017 26th IEEE International Symposium on Robot and Human Interactive Communication (RO-MAN), Aug 2017, Lisbonne, Portugal. 10.1109/ROMAN.2017.8172311 . hal-01692664

\section{HAL Id: hal-01692664 https://hal.science/hal-01692664}

Submitted on 20 Feb 2018

HAL is a multi-disciplinary open access archive for the deposit and dissemination of scientific research documents, whether they are published or not. The documents may come from teaching and research institutions in France or abroad, or from public or private research centers.
L'archive ouverte pluridisciplinaire HAL, est destinée au dépôt et à la diffusion de documents scientifiques de niveau recherche, publiés ou non, émanant des établissements d'enseignement et de recherche français ou étrangers, des laboratoires publics ou privés. 


\title{
The Influence of Individual Social Traits on Robot Learning in a Human-Robot Interaction
}

\author{
Hakim Guedjou $^{1}$, Sofiane Boucenna ${ }^{2}$, Jean Xavier ${ }^{1,3}$, David Cohen ${ }^{1,3}$, Mohamed Chetouani ${ }^{1}$
}

\begin{abstract}
Interactive Machine Learning considers that a robot is learning with and/or from a human. In this paper, we investigate the impact of human social traits on the robot learning. We explore social traits such as age (children vs. adult) and pathology (typical developing children vs. children with autistic spectrum disorders). In particular, we consider learning to recognize both postures and identity of a human partner. A human-robot posture imitation learning, based on a neural network architecture, is used to develop a multi-task learning framework. This architecture exploits three learning levels : 1) visual feature representation, 2) posture classification and 3) human partner identification. During the experiment the robot interacts with children with autism spectrum disorders (ASD), typical developing children (TD) and healthy adults. Previous works assessed the impact on learning of these social traits at the group level. In this paper, we focus on the analysis of individuals separately. The results show that the robot is impacted by the social traits of these different groups' individuals. First, the architecture needs to learn more visual features when interacting with a child with ASD (compared to a TD child) or with a TD child (compared to an adult). However, this surplus in the number of neurons helped the robot to improve the TD children's posture recognition but not that of children with ASD. Second, preliminary results show that this need of a neurons surplus while interacting with children with ASD is also generalizable to the identity recognition task.
\end{abstract}

\section{INTRODUCTION}

During social interactions, complex behavioral and physiological processes occur. Among them, inter-brain synchronization of alpha-mu bands between the right centro-parietal regions (areas involved in social interaction [1]) has been shown to emerge during hand movements coordination [2]. These coupling processes are necessarily impacted by the intrinsic characteristics of individuals. For example, synchrony, either at behavioral or neural level, has been shown to be the physical support of implicit individual traits such as social anxiety [3]. Following an external assessment of human-robot interactions (HRI), Walters et al. showed that distance between individuals and robot, personal social zones, are modified according to individuals' traits such as proactivness[4].

All these works clearly show that the structure of interaction is modified by individuals' social traits. Recently, we introduced a robot-learning-centered approach in which

\footnotetext{
1 Institut des Systèmes Intelligents et de Robotique, Sorbonne Univ., UPMC Univ. Paris 06, UMR7222, F75005 Paris, France guedjoulisir.upmc. fr

2 Équipes Traitement de l'Information et Systèmes, CNRS UMR8051, ENSEA, UCP, 95000 Cergy-Pontoise, France

3 Service de Psychiatrie de l'Enfant et de l'Adolescent, GH PitiéSalpêtrière, APHP, Paris, France
}

we quantify these modifications and evaluated them on the performance of robot to learn with different groups: healthy adults, TD children and children with ASD [5][6]. Using metrics assessing the learning, such as complexity of models, convergence and recognition scores, we show the nature of the group has an impact on robot learning.

We consider a robot multi-task learning for posture imitation and identity recognition. Within this context, imitation is an interesting and focused task that has been investigated in several domains such as robotics and developmental psychology [7]. It is considered as a precursor of social referencing [8]. For example, Meltzoff and Decety suggest that imitation provides an innate foundation for social cognition in infants and underlie the development of theory of mind and empathy for others [9]. Meltzoff also suggests a strong coupling between early imitation in children and the emergence of an identity retrieval function [10].

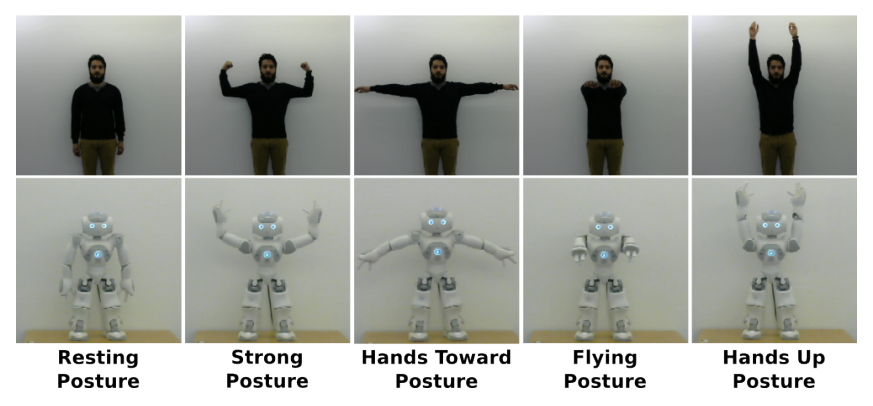

Fig. 1. The robot and the human partner producing the five postures

To effectively learn to imitate posture (see Figure 1) and recognize the identity of individuals, we employed a neural network architecture based on a sensory-motor association paradigm [11]. The architecture performs visual features learning, posture classification and a partner identity recognition. The experiment requires two phases. 1) During the imitation phase, the human imitates the robot while the robot performs body postures. At the same time, learning posture classification requires to map the visual perception stimulus and the proprioception stimulus (posture represented by robot's motor position) [12]. Partner identification exploits a novelty detection to identify new partners and learn their corresponding visual features. 2) During the testing phase, the robot is able to correctly imitate the human performing postures. In our previous works, we demonstrated the efficiency of the robot-learningcentered approach to learn postures imitation, and identity recognition. We also showed that the system was able to 
differentiate between three distinct groups: children with autism spectrum disorder (ASD), typical developing children (TD) and healthy adults demonstrating the validity of the model in terms of partners social assessment [5][6]. By using a neuro-dynamical system, Murata et al. employed a similar approach to observe differences on the structure of interactions according to different partners [13]. However, all these research works did not focus on how individual social traits influence learning processes. In this paper we consider both inter- and intra-group analysis and learning processes. In particular, we assess the interplay between posture recognition, partner identification and individuals' social traits. We also assess the impact of learning parameters such as the number of interactions required to learn termed learning-time and thresholds parameters conditioning the sensitivity of the novelty detection.

This paper is organized as follows. In section II, we describe specific characteristics of the participants, the experimental protocol for the imitation game and the learning architecture that has been employed for posture and identity recognition. We also present the metrics used to evaluate the impact of individual social traits. In section III we report results of the analyses using such metrics to characterize social traits. Finally, in section IV we discuss the impact of participants' characteristics on the robot's learning and we expose the study limitation's.

\section{MATERIALS AND METHODS}

\section{A. Participants}

The experiment included individuals from three distinct groups (see TABLE I): 15 children with ASD, 15 TD children, and 11 adults. Children with ASD were enrolled in the day-care setting for ASD of the Pitié-Salpêtrière hospital. The psychiatric assessment and the parental interview were conducted by two child psychiatrist/psychologists specialized in autism (D. Cohen \& J. Xavier). Assessments included the Autism Diagnostic Interview-revised (ADI-R), the Wechsler Intelligence Scale for Children (fourth version revised, WISC-4R) or equivalent, and the Global Assessment Functioning (GAF) score. The TD children were recruited from several schools in the Paris area. They were chosen according to their developmental ages and genders to match those of the children with ASD. The developmental ages were calculated using the WISC-4R. Adults were students from engineering or medical schools. All the participants from the three groups performed the experiment only one time. The protocol was approved by the Pitié-Salpêtrière hospital ethics committee (Comité de Protection des Personnes). All the participants or parents received information on the experiment and gave written consent before their participation or the participation of their child.

\section{B. Experimental Protocol}

Figure 2 describes the imitation experimental protocol, which is an imitation task with two phases: (a) learning: a human imitating the robot's posture while the robot is

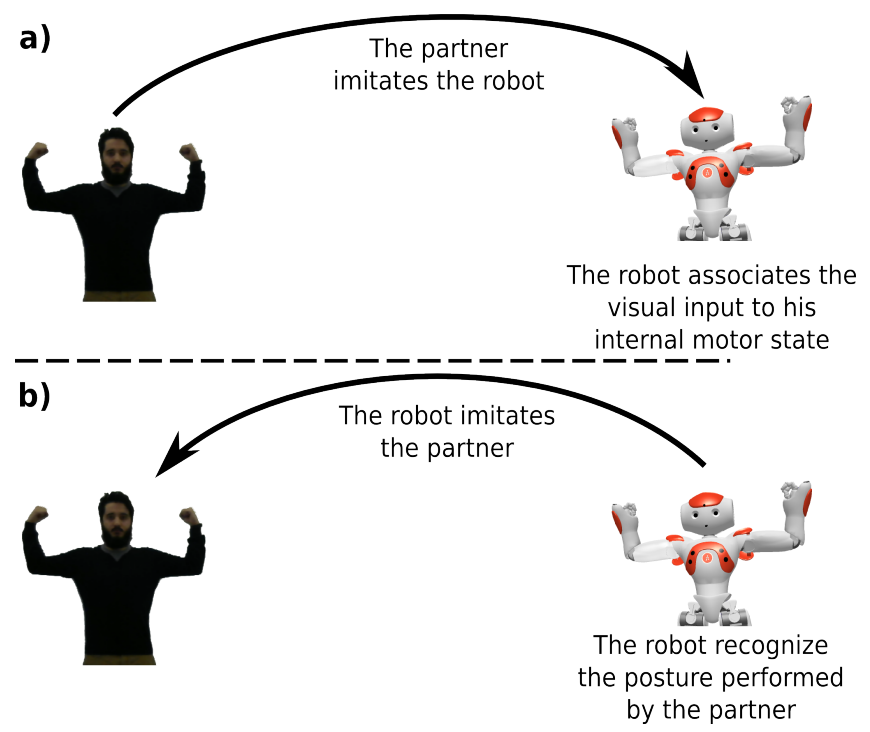

Fig. 2. Overview of the experimental protocol: The robot is the leader in the learning phase (a) then during the test phase (b) the human becomes the leader

learning the mapping between visual representation of human posture and robot's posture, (b) testing: the human is performing postures and the robot is recognizing them and performing them. During the first phase, the robot randomly performs one of the predefined postures (see Figure 1) and the human imitates it. One of the major advantages of this approach is that the robot is able to learn, in real-time, a visual representation of the posture and label it. The outcome of this phase is a computational model able to map a human posture to a posture label. The robot is then able to reproduce it during the validation phase. As most of interactive machine learning approaches, the performance of the model highly depends on the human skills. In this paper, we will specifically address this issue by analyzing several learning and testing schemes while involving partners from different populations.

\section{Architecture}

In this study, we use the same neural network architecture described in our previous work [6], which is based on a sensory motor architecture allowing to learn perceptionaction mapping [11]. During the learning phase the robot performs a random posture according to the robot internal state (RIS) (see Fig. 3). Each internal state of the robot corresponds to a posture (see Fig. 1). In the same time the robot computes a visual representation of the visual input and associates it to the performed posture [12]. In [6], we extended this architecture to not only learn to recognize human postures but also identity of the partner. Figure 3 describes this multi-task learning architecture that exploits (i) visual representation (VF) of human posture and robot posture to further build posture recognition model (PR), (ii) dynamics of the visual representation (VF) to build an identity recognition model (IR). 


\begin{tabular}{|l|c|c|c|}
\cline { 2 - 4 } \multicolumn{1}{c|}{} & ASD $(\mathrm{N}=15)$ & TD $(\mathrm{N}=15)$ & Adults $(\mathrm{N}=11)$ \\
\hline Age, mean $( \pm \mathrm{SD})$, year & $9.25( \pm 1.82)$ & $8.06( \pm 2.49)$ & $25( \pm 3.49)$ \\
Male - Female & $13-5$ & $9-6$ & $7-5$ \\
\hline ADI-R, current, mean $( \pm \mathrm{SD})^{*}$ & $10.77( \pm 5.3)$ & Not relevant & Not relevant \\
Social impairment score & $7.72( \pm 4.22)$ & Not relevant & Not relevant \\
Verbal communication score & $4.3( \pm 3.5)$ & Not relevant & Not relevant \\
Non verbal communication score & $2.5( \pm 1.88)$ & Not relevant & Not relevant \\
Repetitive interest score & $3.3( \pm 1.5)$ & Not relevant & Not relevant \\
Developmental score & $31.1( \pm 5.46)$ & & \\
Total score & \multicolumn{2}{|c}{} \\
\hline ADI-R, 4-5 years, mean $( \pm \mathrm{SD})^{*}$ & $17.33( \pm 8.47)$ & Not relevant & Not relevant \\
Social impairment score & $13.75( \pm 5.72)$ & Not relevant & Not relevant \\
Communication verb score & $8.08( \pm 4.4)$ & Not relevant & Not relevant \\
Communication non-verb score & $5.25( \pm 3.52)$ & Not relevant & Not relevant \\
Repetitive interest score & $3.83( \pm 1.47)$ & Not relevant & Not relevant \\
Developmental score & $48.25( \pm 7.34)$ & & \\
Total score & $73( \pm 14)$ & All controls $>80$ & All controls $>80$ \\
IQ* & $40.27( \pm 9.44)$ & All controls $>90$ & All controls $>90$ \\
GAF score & \multicolumn{2}{|c}{} \\
\hline
\end{tabular}

TABLE I

PARTICIPANTS' CLINICAL ASSESSMENTS RESUlTS :

\begin{abstract}
*The Autism Diagnostic Interview-Revised is a parental interview to score autism core symptoms as they are presenting currently and also as the presented retrospectively when the child was aged 4-5 years. The difference between current and 4-5 year scores indicates that the children recruited for this experiment improved with age most symptomatic dimensions
\end{abstract}

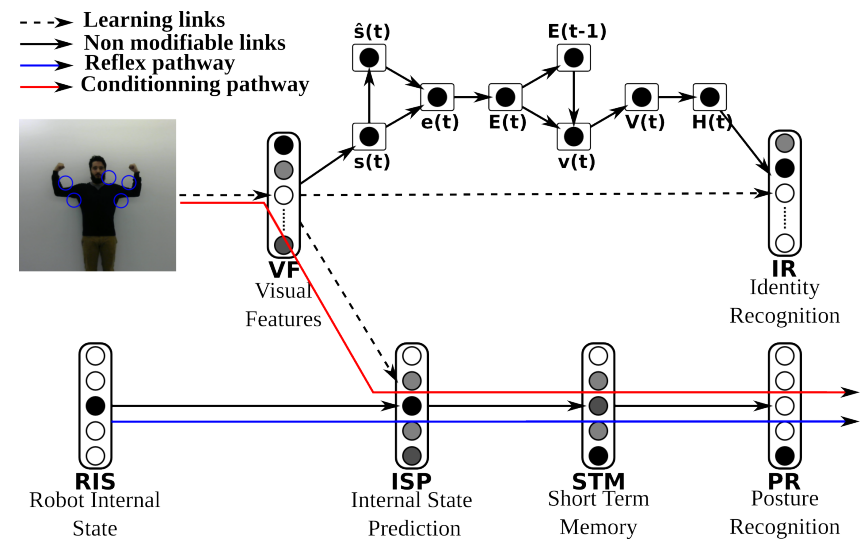

Fig. 3. Sensory motor architecture allowing the imitation and partner recognition tasks

1) Visual processing system: Unlike many HRI systems, this model does not use a framing step to constraint on how and where the visual features are extracted. The visual features detection is based on an attentional vision mechanism, working on a sequential exploration of saliency points in the image. We begin by performing a gradient extraction on a gray scale image, a convolution between the norm of the gradient and a difference of Gaussians (DOG) is then performed. A competition in the result provides the most active points as focus points. For each focus point a descriptor/feature is extracted. This descriptor consists on a log-polar transformation of a local area around the focus point. This transformation makes the descriptor robust to rotation and distance.

2) Visual features learning: The extracted descriptors are learned and recognized by the VF group fo neurons (Visual features). VF is a $\mathrm{k}$-means variant allowing the modification of the number of neurons [14] called SAW (selective adaptive winner). The output of VF is computed as:

$$
V F_{j}=\text { net }_{j} \cdot H_{\gamma} \text { with net }_{j}=1 \frac{1}{N} \sum_{i=1}^{N}\left|W_{i j}-I_{i}\right|
$$

$V F_{j}$ is the activity of neuron $\mathrm{j}$ in the $V F$ group. $N$ is the size of the vector that represents one visual feature pattern I. $H_{\gamma}$ is the Heaviside function:

$$
H_{\gamma}=\left\{\begin{array}{l}
1 \text { if } \gamma<x \\
0 \text { otherwise }
\end{array}\right.
$$

$\gamma$ is a vigilance parameter employed as a threshold for descriptor recognition. When the descriptor recognition is below $\gamma$, then a new neuron is recruited (incremental learning). This model enables the recruitment of neurons to adapt to the dynamics of the visual input. The learning rule allows both one-shot learning and long-term averaging. The modification of the weights $W i j$ is computed as follows :

$$
\begin{gathered}
\Delta W_{i j}=a_{j}(t) I_{i}+\mu\left(1-\delta_{j}^{k}\right)\left(I_{i}-W_{i j}\right)\left(1-V F_{j}\right) \\
k=\operatorname{ArgMax}\left(a_{j}\right) \text { and } a_{j}=\left\{\begin{array}{l}
1 \text { if new neuron } \\
0 \text { otherwise }
\end{array}\right.
\end{gathered}
$$

$\delta_{j}^{k}$ is the Kronecker symbol :

$$
\delta_{j}^{k}=\left\{\begin{array}{l}
1 \text { if } j=k \\
0 \text { otherwise }
\end{array}\right.
$$

The $\mu$ value allows a long-term averaging through descriptors. During the recruitment of a neuron the weights are modified to correspond to the descriptor $\left(a_{j}(t)=1\right)$. If a neuron fires to a given descriptor the weights of the neuron are averaged to get closer to this local-view $\left(\mu\left(I_{i}-W_{i j}\right)(1-\right.$ $\left.V F_{j}\right)$ ). The closer the descriptor's pattern is from the weights 
of the neuron the less the weights are modified and vice versa. The $\mu$ plays a main role in the recognition task : if $\mu$ is too high the memory used is higher and the generalization of the system decreases but if $\mu$ is too low the averaging will be too high therefore the recognition scores will reduce.

3) Posture learning and prediction: For the posture learning, we use a clustering approach. We classify postures according to the activation of VF's neurons. Each RIS neuron (Robot Internal State) corresponds to a posture. During the learning phase, the ISP's group of neuron (Internal State Prediction) links the simultaneously activated VF's neurons and RIS's neuron. Considering a reflex pathway this model builds a conditioning mechanism that associates each posture's corresponding VF's neurons to a corresponding ISP's neuron. The ISP model is based on a Widrow and Hoff rule [15]. It requires enough relevant descriptors on each image to learn it correctly. The learning follows the least mean square (LMS) rule :

$$
\Delta w_{i j}=\mu \cdot V F_{i} \cdot\left(R I S_{j}-I S P_{j}\right)
$$

4) Final filtering: To avoid real-time problems during the in-line HRI on testing phase, a filtering mechanism is used. This mechanism consists on an averaging of the ISP's neurons activation over time ( $\mathrm{N}$ iterations). It is computed as follows:

$$
S T M_{i}(t+1)=\frac{1}{N} I S P_{i}(t+1)+\frac{N-1}{N} S T M_{i}(t)
$$

Finally the MP group uses a winner takes all method on the STM result to recognize the posture which will be performed by the robot. To avoid partner reaction time problems during this experiment, after a change of of posture, we do not exploit the first frames for learning. This approach allows to reduce the impact of transitions between postures.

5) Novelty detector: The novelty detector aims at identifying significant changes in the dynamics of the VF neural network. We compare the dynamics of $\mathrm{VF}$ to its average prediction. The novelty detection is computed by an analysis of the prediction error of the number of neurons in VF. The error $e(t)$ is computed as the difference between the predicted number of neurons on $\mathrm{VF} \hat{s}(t)$ and the actual real number $s(t)$ :

$$
e(t)=\hat{s}(t)-s(t)
$$

Then we calculate the mean error $E(t)$

$$
E(t)=\frac{1}{N} \sum_{t_{i}=1}^{N} e\left(t-t_{i}\right)
$$

Finally we perform a mean gradient of the resulted signal:

$$
V(t)=\frac{1}{M} \sum_{t_{i}=1}^{M}-\frac{\delta E\left(t-t_{i}\right)}{\delta\left(t-t_{i}\right)}
$$

A threshold is used to compute a rising edge detection on $V(t)$. The rising edge signal $H(t)$ is correlated to novelty. When novelty occurs, a new neuron is recruited in the IR group of neurons (Identity Recognition) and associates the neurons in VF to this new identity. This threshold is directly connected to the sensitivity of the novelty detection. Here, we use the same learning model as in the ISP group of neurons.

\section{Metrics}

The aim of this study is to investigate the influence of partners' specificities on a robot learning. Each learning level of this architecture provides different metrics and they potentially provide insights on this impact. First, the VF group recruits neurons to learn descriptors corresponding to postures. This VF number of neurons can be considered as a measure of learning complexity and variability of posture imitation of the partner. Second, to evaluate the learning by imitation task as a whole, we use the posture recognition score. The third metric is the number of recruited neurons in the IR neural network while variating the sensitivity of the novelty detector by variating the threshold. And finally, we use the participant recognition score as a metric of the identity recognition task.

\section{E. Evaluation protocols}

The robot interacted with each participant (see Sec IIA) following the protocol described in section II-B. During this interaction a database is built for an off-line processing and analysis. The robot records all images and annotates theme according to its posture. The learning phase images are well labeled since the human imitate robot's postures. The validation phase images are labeled according to the robot posture recognition task (see Fig 1)

To evaluate the multi-task learning neural architecture, we proposed several specific evaluation protocols. In the three first evaluation protocols we evaluate the posture recognition task, therefore it is necessary to have a data base with a perfect posture labelisation. Consequently we use only the images recorded from the learning phase which are divided in two (learning images, validation images). In the last evaluation protocol, we evaluate how the identity recognition task is impacted by the posture labelisation of the database. Therefore we are using all the database which is divided in two separate sets: learning images, validation images. In this last evaluation protocol we need to have the same number of participants in the three groups to have the same identity recognition probabilities. Therefore 4 children have been discarded from the TD and ASD groups randomly

1) Protocol 1: Group specific interaction analysis: In this first analysis, we reproduce off-line the interaction of the robot with 41 people from the different groups: 15 ASD children, 15 TD children and 11 Adults). Each interaction is independent from the others. Each partner's interaction has the same learning time, approximately 1 minute corresponding to 155 frames. We then analyze the results according to each partner's group.

2) Protocol 2: Posture specific analysis: To investigate whether the interaction's results are influenced by the nature of postures, we learned posture specific models (i.e., learning and testing with only one posture). We performed an offline learning/testing for each of the five postures. Each 
posture is performed in total for approximately fourteen seconds corresponding to 33 learned frames per posture for each partner. We then analyze posture specific model results according to the partner's group.

3) Protocol 3: Learning-time impact on posture recognition scores: The purpose of this protocol is to evaluate the impact of the interaction learning duration on the posture recognition scores for each group. In other words, we evaluate the impact of exposure of the model to postures. We modify off-line the learning-time from two seconds to 50 seconds (corresponding respectively to a variation from 1 frame to 115 frames for each posture). Then for each learning time, we evaluate posture recognition score for each partner of each group and we also analyze the results by groups (TD children, children with ASD, adults).

4) Protocol 4: Novelty detection threshold impact on identity recognition scores: In this protocol, to have the same amount of subjects in each group, we randomly discarded 4 children in the TD and ASD groups. The resulting database was composed of 11 children with ASD, 11 TD children and 11 adults. We simulated a variation of the threshold parameter controlling the novelty detection sensitivity. The partners' identities in each group are learned sequentially. Then, we evaluate the identity recognition scores for each group (TD children, children with ASD, adults) and the number of neurons used to learn the identities.

\section{EXPERIMENTAL RESULTS}

Each result of this section corresponds to an aforementioned protocol.

\section{A. Result 1: Group specific interaction analysis}

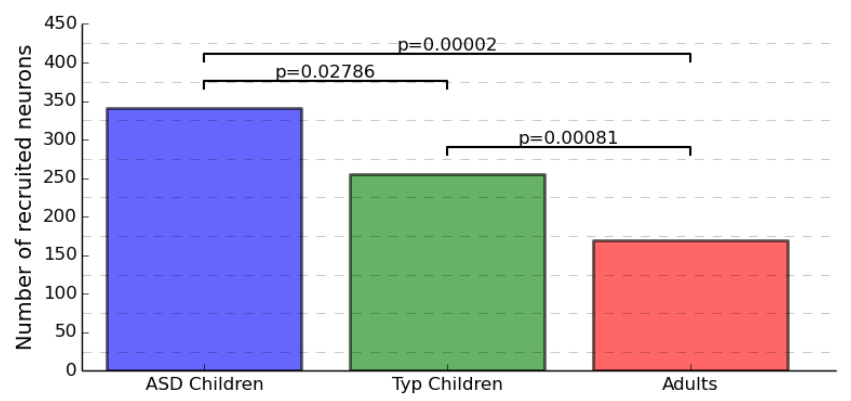

Fig. 4. Mean number of recruited neurons in the VF group of neurons during the learning phase for each group (ASD, TD, Adults)

In Fig. 4, the number of recruited neurons needed to learn in the VF neural network for each partner are reported. We present the mean number by group (ASD, TD, Adults). The neurons needed to learn for each group significantly differ (mean $\mathrm{ASD}=341$, mean $\mathrm{TD}=255$, mean adult $=169$ ). This results describes the fact that the interaction with an ASD child is more complex. On average, the architecture needs twice as many neurons to learn with an ASD child than for an interaction with an adult and one-third more neurons than for an interaction with a TD child. To test whether samples originate from the same distribution we used the KruskalWallis test, $p<0.05$.

\section{B. Result 2: Posture specific analysis}

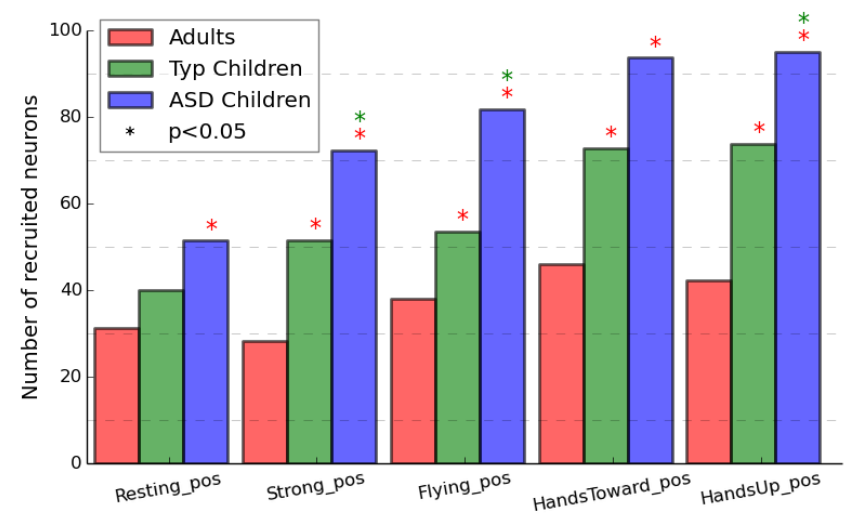

Fig. 5. Mean number of neurons needed in the VF group of neurons during the learning phase for each group (ASD, TD, Adults) according to each posture

To assess whether or not the previous result is similar across all the postures, we performed a posture specific analysis. First, we find that the number of recruited neurons in the VF neural network differs according to the postures. The ranking of the postures in terms of recruited neurons number is the same across the three groups. Concerning the inter-group evaluation, there is a confirmation of Fig.4 independently of the postures. For all the postures, it requires two times more neurons to interact with a child with ASD than to interact with an adult except for the resting posture where this ratio decreases. All the comparisons are significant (Kruskal-Wallis test $: p<0.05$ ) except for the resting posture where the comparisons TD vs. ASD and TD vs. Adult are not significant.

Using the two previous analyses we calculated the similarity between the different postures in terms of descriptors. This similarity is computed by comparing the number of recruited neurons in Fig. 4 and the summation of the number of neurons of the different postures for each group in Fig.5. When postures are learned sequentially, similar descriptors are learned only one time. However, when postures are learned separately, similar descriptors are learned for each posture. The results showed that adults partners have less similarity in their different postures than children partners.

\section{Result 3: Learning-time impact on posture recognition scores}

Interacting with a child with ASD requires more VF neurons than with a partner of the two other groups. To investigate if this surplus of neurons improves the posture recognition we compared the results of posture recognition for each partner of each group while changing the learningtime The analysis shows that the longer is the interaction the better is posture recognition performance for a given partner. We also find a strong correlation between the number of recruited VF neurons and the recognition scores for each group: the correlation is higher than 0.95 with a $p$-value $<$ $10^{-12}$ for each group (Spearman correlation test). However, 


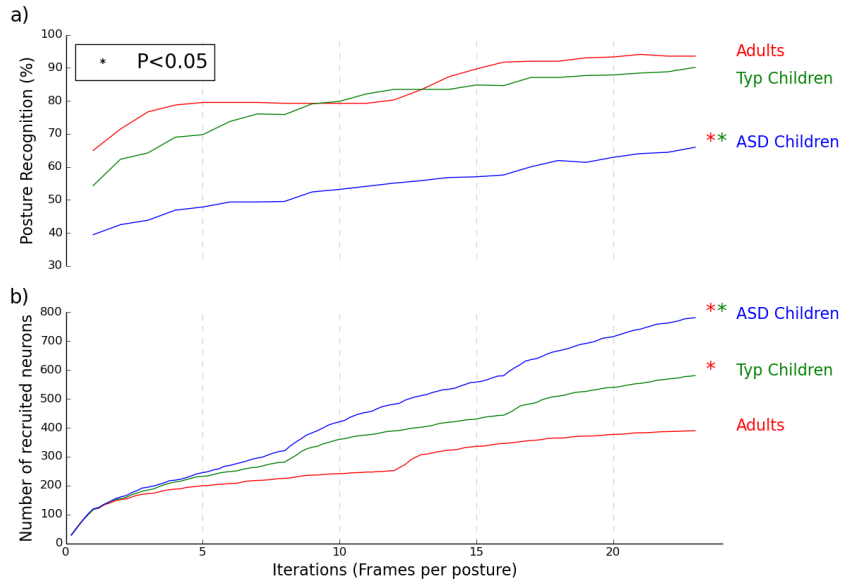

Fig. 6. Correspondence through time between a) the mean percentage for each group of the recognition of each individuals postures and b) The mean number of recruited neurons for each group when the robot is learning with one individual

even if interacting with children with ASD requires a higher number of VF neuron recruitment, their posture recognition is lower than that of the two other groups. In contrast, TD's neuron number increase compensates the difference with adults in terms of posture recognition scores. In graph (a) the comparisons TD vs. ASD and ASD vs. Adult are significant (Kruskal-Wallis test for each iteration).In graph (b) all the comparisons, TD vs. ASD, TD vs. Adult and ASD vs. Adult are significant (Kruskal-Wallis test for each iteration : $p<0.05$ )

\section{Result 4: Novelty detector threshold impact on learning according to each group}

In previous works [6] the threshold parameter have been arbitrary chosen to allow one neuron recruitment per partner. In this section we analyze the impact of this parameter on identity recognition for the three groups. We also evaluate the number of neurons recruited in the IR neural network for each group (ASD, TD, adults) to assess how this learning level is modifier in each group. We also asses the identity recognition scores for each group while variating the threshold parameter. Adult partners recognition score is higher than TD children one. TD children score is higher compared with children with ASD for all the tested thresholds. Considering the recruitments of neurons, TD children and children with ASD require a threshold $\leq 36 \times 10^{-4}$ and adults require a threshold $\leq 41 \times 10^{-4}$ otherwise the system recruits less than 11 neurons to learn the 11 partners which means that the system is confusing between some people. For almost all the threshold intervals, the system recruits more neurons while learning from children with ASD compared to TD children and from TD children compared with adults. As we can expect there is a strong correlation between the IR number of neurons and the identity recognition scores for the three groups a (Spearman correlation : $\mathrm{ASD}=0.77, \mathrm{TD}=0.92$, adult $=0.54)$ The system is able to recognize adults partners even with a low amount of neurons (12 neurons for a score

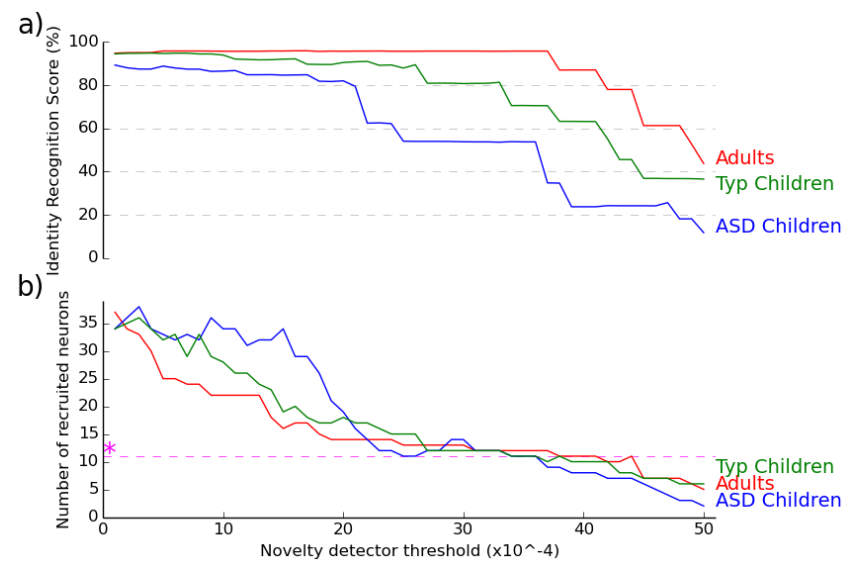

Fig. 7. Correspondence through variation of the novelty detection threshold between a) the identity recognition score for each group and b) The number of recruited neurons by the IR group of neurons to code 11 partners for each group. *Represents the minimum number of neurons possible for the identity recognition task. Less than 11 neurons for the 11 partners means the system makes a confusion between some partners

of $96 \%, 11$ neurons for $87 \%)$. For TD children it needs more (38 neurons for a score of 95\%, 14 neurons for $88 \%$ ). Even when ASD recruits more neurons, their recognition scores are the lowest. When the system recruits less neurons with children with ASD we note that the identity recognition score for people of that group decreases under $80 \%$ and then under $60 \%$.

\section{DISCUSSION \& CONCLUSIONS}

In this paper, we used a neural architecture to simultaneously learn partner posture and identity recognition. The aim was to evaluate the impact of partners social traits (ASD vs. $\mathrm{TD}$, children vs. adults) on robot learning. While the human imitates the robot, the VF neural network (visual features) recruits neurons to learn the visual inputs of the robot. The ISP neural network (Internal state prediction) associates the VF's neurons to the RIS neurons (Robot Internal State). The IR neural network (Identity Recognition) recruits neurons to learn partners identity thanks to a coupling between the VF numbers of neurons needed to learn and a novelty detector. In this paper we generalized the robot centered approach to an interaction with individuals (posture recognition) and a second group task (identity recognition). The results shown in this paper support our view that the complexity of the partners behavior can be detected in a learning system.

Previous results [5] showed that the robot learns more visual features while interacting with a group of children with ASD than it does when interacting with a group of TD children or with an group of adults. Results in this paper show that the same aspect is also perceivable when interacting with individuals of these different groups. the architecture needs more visual features when interacting with a child with ASD (compared to a TD child) or with a TD child (compared to an adult). Furthermore this results show that for all partners of the different groups there was a correlation between the learning phase duration, the number 
of recruited neurons and the posture recognition scores. However, while the surplus of recruited neurons in VF for the TD partners ameliorates consistently the posture recognition score to reach adults scores, the surplus for partners with ASD is not sufficient. This results shows that individuals with ASD have a higher variability in terms of posture realization and the architecture has more difficulties to capture this variability.

In this paper we variated an important parameter in our architecture which have been fixed in previous works. The novelty detector threshold represents the sensitivity of the identity recognition group of neurons (IR) to detect new partners and learn to recognize theme. This experiment's result showed that the recruitment of neurons behavior in the posture recognition task is generalizable to the identity recognition one, the system recruits more neurons while learning with a child with ASD (compared to a TD child) or with a TD child (compared to an adult). To achieve the identity recognition task and reach score higher than $85 \%$ The architecture needs 12 neurons for adults, 15 neurons for TD children and more than 30 for children with ASD. This can be considered as an adaptation of the system to the complexity and variability in the partners' behavior.

The current study has several limitations: (1) the group were large enough to allow learning but small to conduct statistical analysis to correlate learning scores and clinical characteristics in the ASD group. (2) Ideally to assess both diagnosis and age, we should also perform the experiment with a group of adult individuals with ASD. (3) The imitation process with Nao included some form of discontinuity with poses between each change of motor posture. We wonder whether the use of a more dynamic imitation interaction with an avatar acting like a tightrope walker would produce different forms of learning results.

\section{ACKNOWLEDGMENT}

The work received fundings from ROMEO2 and Labex SMART (ANR-11-LABX-65) supported by French state funds managed by the ANR within the Investissements dAvenir programme under reference ANR-11-IDEX-000402 .

\section{REFERENCES}

[1] A. Perry, N. F. Troje, and S. Bentin, "Exploring motor system contributions to the perception of social information: Evidence from eeg activity in the mu/alpha frequency range," Social Neuroscience, vol. 5, no. 3, pp. 272-284, 2010.

[2] G. Dumas, J. Nadel, R. Soussignan, J. Martinerie, and L. Garnero, "Inter-brain synchronization during social interaction," PloS one, vol. 5, no. 8, p. e12166, 2010.

[3] K. Yun, K. Watanabe, and S. Shimojo, "Interpersonal body and neural synchronization as a marker of implicit social interaction," Scientific reports, vol. 2, p. 959, 2012.

[4] M. L. Walters, K. Dautenhahn, R. Te Boekhorst, K. L. Koay, C. Kaouri, S. Woods, C. Nehaniv, D. Lee, and I. Werry, "The influence of subjects' personality traits on personal spatial zones in a human-robot interaction experiment," in Robot and Human Interactive Communication, 2005. ROMAN 2005. IEEE International Workshop on. IEEE, 2005, pp. 347-352.
[5] S. Boucenna, S. Anzalone, E. Tilmont, D. Cohen, and M. Chetouani, "Learning of social signatures through imitation game between a robot and a human partner," IEEE Transactions on Autonomous Mental Development, vol. 6, no. 3, pp. 213-225, Sept 2014.

[6] S. Boucenna, D. Cohen, A. N. Meltzoff, P. Gaussier, and M. Chetouani, "Robots learn to recognize individuals from imitative encounters with people and avatars," Scientific Reports, vol. 6, 2016.

[7] A. N. Meltzoff, P. K. Kuhl, J. Movellan, and T. J. Sejnowski, "Foundations for a new science of learning," science, vol. 325, no. 5938, pp. 284-288, 2009.

[8] S. Boucenna, P. Gaussier, and L. Hafemeister, "Development of first social referencing skills: Emotional interaction as a way to regulate robot behavior," IEEE Transactions on Autonomous Mental Development, vol. 6, no. 1, pp. 42-55, 2014.

[9] A. N. Meltzoff and J. Decety, "What imitation tells us about social cognition: a rapprochement between developmental psychology and cognitive neuroscience," Philosophical Transactions of the Royal Society of London B: Biological Sciences, vol. 358, no. 1431, pp. 491-500, 2003.

[10] A. N. Meltzoff and M. K. Moore, "Early imitation within a functional framework: The importance of person identity, movement, and development," Infant behavior \& development, vol. 15, no. 4, pp. 479-505, 101992.

[11] P. Gaussier and S. Zrehen, "Perac: A neural architecture to control artificial animals," Robotics and Autonomous Systems, vol. 16, no. 2 , pp. 291-320, 1995.

[12] S. Boucenna, P. Gaussier, P. Andry, and L. Hafemeister, "A robot learns the facial expressions recognition and face/non-face discrimination through an imitation game," International Journal of Social Robotics, vol. 6, no. 4, pp. 633-652, 2014.

[13] S. Murata, K. Hirano, H. Arie, S. Sugano, and T. Ogata, "Analysis of imitative interactions between humans and a robot with a neurodynamical system," in System Integration (SII), 2016 IEEE/SICE International Symposium on. IEEE, 2016, pp. 343-348.

[14] T. Kanungo, D. M. Mount, N. S. Netanyahu, C. D. Piatko, R. Silverman, and A. Y. Wu, "An efficient k-means clustering algorithm: Analysis and implementation," IEEE transactions on pattern analysis and machine intelligence, vol. 24, no. 7, pp. 881-892, 2002.

[15] B. Widrow, M. E. Hoff, et al., "Adaptive switching circuits," in IRE WESCON convention record, vol. 4, no. 1. New York, 1960, pp 96-104. 